\title{
Comparison of echocardiographic indices of right ventricular systolic function and ejection fraction obtained with continuous thermodilution in critically ill patients
}

\author{
Romain Barthélémy ${ }^{1,4^{*}}$ D, Xavier Roy ${ }^{1}$, Tujia Javanainen ${ }^{1,2}$, Alexandre Mebazaa ${ }^{1,2,3}$ and \\ Benjamin Glenn Chousterman ${ }^{1,2,3}$
}

\begin{abstract}
Background: Though echocardiographic evaluation assesses the right ventricular systolic function, which of the existing parameters best reflects the right ventricular ejection fraction (RVEF) in the critically ill patients is still uncertain. We aimed to determine the relationship between echocardiographic indices of right ventricular systolic function and RVEF.

Methods: Prospective observational study was conducted in a mixed Surgical Intensive Care Unit (Hôpital Lariboisière, Paris, France) from November 2017 to November 2018. All critically ill patients monitored with a pulmonary artery catheter were assessed. We collected echocardiographic indices of right ventricular function (tricuspid annular plane systolic excursion, TAPSE; peak systolic velocity of pulsed tissue Doppler at lateral tricuspid annulus, S'; fractional area change, FAC; right ventricular index of myocardial performance, RIMP; isovolumic acceleration, IVA; end-diastolic diameter ratio, EDDr) and compared them with the RVEF obtained from continuous volumetric pulmonary artery catheter.

Results: Twenty-five patients were analyzed. Admission diagnosis was acute heart failure in 11 patients and septic shock in 14 patients. Median age was 70 years [57-80], norepinephrine median dose was $0.29 \mu \mathrm{g} / \mathrm{kg} /$ min [0.14-0.50], median Sequential Organ Failure Assessment score was 12 [10-14], and mortality at day 28 was $56 \%$. When compared to RVEF, TAPSE had the highest correlation coefficient (rho $=0.78,95 \% \mathrm{Cl} 0.52$ to $0.89, p<0.001$ ). S' was also correlated to RVEF ( $r$ ho $=0.64,95 \% \mathrm{Cl} 0.60$ to $0.80, p=0.001$ ) whereas FAC, RIMP, IVA, and EDDr did not. TAPSE lower than $16 \mathrm{~mm}$, S' lower than $11 \mathrm{~cm} / \mathrm{s}$, and EDDr higher than 1 were always associated with a reduced RVEF.
\end{abstract}

Conclusions: We found that amongst indices of right ventricular systolic function, TAPSE and $S^{\prime}$ were well correlated with thermodilution-derived RVEF in critically ill patients.

Keywords: Critical illness, Ventricular dysfunction, Right, Catheterization, Swan-Ganz, Thermodilution, Echocardiography, 2D, Echocardiography, Doppler

\footnotetext{
* Correspondence: romain.barthelemy@aphp.fr

'Department of Anaesthesia and Critical Care, Lariboisière Hospital, DMU

Parabol, APHP.Nord, Paris, France

${ }^{4}$ Réanimation Chirurgical Polyvalente, Hôpital Lariboisière, 2 rue Ambroise

Paré, 75475 Paris Cedex 10, France

Full list of author information is available at the end of the article
}

(c) The Author(s). 2019 Open Access This article is distributed under the terms of the Creative Commons Attribution 4.0 International License (http://creativecommons.org/licenses/by/4.0/), which permits unrestricted use, distribution, and reproduction in any medium, provided you give appropriate credit to the original author(s) and the source, provide a link to the Creative Commons license, and indicate if changes were made. The Creative Commons Public Domain Dedication waiver (http://creativecommons.org/publicdomain/zero/1.0/) applies to the data made available in this article, unless otherwise stated. 


\section{Background}

Right ventricular (RV) dysfunction is frequently encountered in intensive care unit (ICU) and has been associated with poor outcome in many acute clinical situations such as respiratory failure, septic shock, acute heart failure, pulmonary embolism, myocardial infarction, and acute decompensated pulmonary hypertension [1-3]. It occurs when RV preload and/or afterload are increased and may lead to acute right ventricular failure with systemic congestion and/or low cardiac output. However, this syndrome has no consensual definition, and its diagnosis is mostly based on a complex association of context, clinical presentation, and echocardiographic evaluation [1, 3]. The gold standard for measurement of $\mathrm{RV}$ systolic function is considered to be RV ejection fraction (RVEF) measured by cardiac MRI [2], but the use of this technique is impractical in hemodynamically impaired critically ill patients requiring mechanical ventilation and continuous infusion of inotropes or vasopressors.

According to international consensus, transthoracic echocardiography (TTE) is the first-line recommended exam when investigating the hemodynamic failure of ICU patients [4]. International societies of echocardiography recommend to assess RV systolic function with several indices [5]. Some of the proposed indices have been compared with cardiac MRI [6-8]. However, none have been validated with the RVEF in ICU patients [9], and which of those parameters best reflects RVEF in the critically ill is uncertain.

To investigate this question, we compared echocardiographic indices of RV systolic function to RVEF measured by continuous volumetric pulmonary artery catheter (PAC). This method is not the gold standard for RVEF measurement and was chosen as the best bedside method available that is not based on echocardiography.

\section{Material and methods}

This is a prospective observational study aiming to compare TTE indices of RV systolic function to RVEF measured by continuous volumetric PAC. This study was approved by an institutional ethics committee (Comité d'éthique de la Société Française d'Anesthésie-Réanimation, IRB 00010254-2016-034) which waived the need of signed informed consent.

\section{Patients}

The study was conducted between November 2017 and November 2018 in a 20-bed mixed surgical ICU without post-cardiac surgery patients (Hôpital Lariboisière, Paris, France). Our ICU has a long experience of PAC use. In our ICU protocol, PAC is indicated as according to international consensus, i.e., in case of refractory shock not responding to initial therapy and suspicion of $\mathrm{RV}$ failure [4]. Postoperative cardiac surgery or scheduled PAC monitoring is not referred to our ICU.

Inclusion criteria were all consecutive patients in whom a PAC had been used for hemodynamic monitoring for less than $24 \mathrm{~h}$.

Exclusion criteria were age $<18$ years old, insufficient TTE quality to allow the measurements of indices of RV systolic function (i.e., inability of the investigator to obtain a regular apical 4-chamber view to allow accurate measurements of ventricle dimensions, and accurate time-motion or Doppler measurements without angle correction), cardiac dysrhythmias responsible of irregular echocardiographic patterns, and parameters known to interfere with continuous volumetric PAC performance: hypothermia (central temperature $<35^{\circ} \mathrm{C}$ ), intracardiac shunt, and tricuspid regurgitation (TR) evaluated after PAC insertion classified as severe according to recommendations [10] (Additional file 1).

\section{Data collection}

Patients' demographic characteristics, diagnosis at admission, hemodynamic status (heart rate, mean arterial pressure, systolic arterial pressure, diastolic arterial pressure, mixed venous oxygen saturation, arterial lactate), current vasopressor and inotrope treatment, settings of mechanical ventilation, Simplified Acute Physiology Score (SAPS II) at admission, current Sequential Organ Failure Assessment (SOFA), comorbidities, Charlson comorbidity index, and mortality at day 28 were collected.

\section{Pulmonary artery catheter}

PAC with continuous cardiac output and volumetric measurement (Swan-Ganz CCOmbo V, Edwards Lifesciences, Irvine, CA, USA) was connected to the bedside monitor for pressure measurement (Intellivue MP70, Philips Electronics Nederland B.V., Eindhoven, The Netherlands). Position was checked on the post-insertion chest X-ray or any other more recent X-ray. Zeroing at the phlebostatic level was performed, and waveform of pulmonary artery pressure (PAP) and right atrial pressure (RAP) checked to ensure good positioning. Quality of the arterial pressure signal was assessed with a fast-flush test ensuring no abnormal signal damping. Criteria for the adequate wedge position of the tip of the catheter were as follows: (1) change of the pulmonary artery waveform to atrial waveform during occlusion, (2) a mean end-expiratory pulmonary capillary wedge pressure (PCWP) lower than the diastolic pulmonary arterial pressure (dPAP), and in case of mechanical ventilation, (3) a ratio of induced variations of PCWP to induced variations of systolic pulmonary arterial pressure (sPAP) lower than $1.5[11,12]$.

Continuous volumetric PAC was also connected to the Vigilance II monitor (Edwards Lifesciences, Irvine, CA, USA) for continuous cardiac output monitoring and 
computation of the RVEF. Briefly, the continuous PAC is supplied with a heating filament located in the right ventricle and a distal thermistor located in the pulmonary artery. The above criteria ensure the correct position of the catheter. A pseudorandom binary heating pattern delivered to the filament and the temperature variation registered by the thermistor are combined by a crosscorrelation algorithm to compute the thermodilution curve every $54 \mathrm{~s}[13,14]$. The largest reported time delay of the device after therapeutic intervention is lower than $20 \mathrm{~min}$; thus, a 20-min stable period without intervention was required before hemodynamic evaluation [14]. During this period, doses of medications and mechanical ventilation settings were not modified, and fluid boluses were avoided. The cardiac output (CO) is determined from the Stewart and Hamilton principle $[15,16]$ adapted to thermodilution by Fegler $[17,18]$. The stroke volume (SV) is computed from the $\mathrm{CO}$ divided by the heart rate obtained from the ECG of the bedside monitor. The ratio of end-systolic volume to end-diastolic volume is derived from the wash-out portion of the thermodilution curve and the heart rate; RVEF can therefore be calculated $[19,20]$.

To ensure concomitant evaluation of both echocardiographic and PAC indices, the following parameters were collected just before the TTE was performed: systolic pulmonary arterial pressure (sPAP), diastolic pulmonary arterial pressure (dPAP), mean pulmonary arterial pressure (mPAP), pulmonary capillary wedge pressure (PCWP), right atrial pressure (RAP), cardiac output (CO), stroke volume (SV), right ventricle end-diastolic volume (RVEDV), right ventricle end-systolic volume (RVESV), and RVEF. Coronary perfusion pressure (CPP) was secondarily calculated as DAP minus RAP.

\section{Transthoracic echocardiography}

Echocardiographic data were obtained from a $1.5-3.6-\mathrm{MHz}$ cardiac probe connected to a Vivid i echograph (GE Healthcare, Wauwatosa, WI, USA). To ensure consistency in the measurements of the echocardiographic indices, all TTE were performed by a limited number of physicians with advanced expertise in critical care echocardiography (R.B., X.R., or T.J.). Before the beginning of the inclusions, they all studied the latest recommendations of the American Society of Echocardiography endorsed by the European Association of Echocardiography [5] and performed several exams all together to harmonize their practices. ETT parameters were measured and calculated off-line on acquired images, blinded from the PAC values, on an average of 3 measurements.

The following RV systolic function parameters were measured as recommended by international guidelines [5]: fractional area change (FAC), tricuspid annular plane systolic excursion (TAPSE) in M-mode, and indices derived from the pulsed tissue Doppler at lateral tricuspid annulus-peak systolic velocity ( $\left.\mathrm{S}^{\prime}\right)$, RV index of myocardial performance (RIMP), and isovolumic acceleration (IVA). RIMP is defined as the ratio of isovolumic time and ejection time; isovolumic time is calculated as the tricuspid opening time minus ejection time. Time interval is measured from a single beat. IVA is defined as the ratio of peak isovolumic myocardial velocity and time to peak velocity. The onset of myocardial acceleration is at the zero-crossing point of myocardial velocity during isovolumic contraction (Additional file 1).

Other RV parameters not directly related to systolic function were also recorded: RV to left ventricle (LV) end-diastolic diameter ratio (EDDr) and peak TR velocity by continuous waved Doppler [1]. EDDr is defined as the ratio of the basal diameter of RV to LV; basal diameter is consensually defined as the maximal short-axis dimension in the basal one third of the right ventricle seen on the 4chamber view [5]. sPAP was calculated as the sum of the TR maximal velocity pressure gradient (according to the simplified Bernoulli equation) and the measured RAP [21]. The following LV parameters were also recorded: LV outflow tract diameter, septum and posterior wall thicknesses, LV diastolic diameter, LV ejection fraction (LVEF), mitral inflow Doppler velocity (E and A waves), pulsed waved tissue Doppler velocity at lateral mitral annulus (e' wave), and velocity-time integral of pulsed waved Doppler at LV outflow tract.

\section{Statistical analysis}

The results are expressed as median [interquartile range] for continuous variables and number (percentage) for categorical variables. Spearman's correlations and 95\% confident intervals (CI) were performed amongst all indices. Missing data were handled by pairwise deletion. Results of correlations are presented in a graphical correlation matrix. Tested variables were ordered using a hierarchical clustering method. A $p$ value less than 0.05 was considered as significant.

Specificity (Sp), sensitivity (Se), positive predictive value (PPV), and negative predictive value (NPV) to detect a reduced RVEF were determined for all indices at the recommended thresholds [5]. 95\% CI were computed with 2000 bootstrap resampling of the receiver operating characteristics curves. Different thresholds for reduced RVEF are proposed in the literature, usually ranging from $50 \%$ (moderately reduced) to $30 \%$ (severely reduced) [22]. We chose a cutoff value of $35 \%$ as proposed by Vanderpool et al. [23] because it is the threshold that best predicts long-term outcomes. We also tested a lower threshold of $25 \%$ to take into account the reported bias of the continuous thermodilution method $[24,25]$. 
All statistical analyses were performed using $\mathrm{R}$ statistical software version 3.4.3 (R Core Team, 2017, R Foundation for Statistical Computing, Vienna, Austria, https://www.Rproject.org). The visualization of the correlation matrix was drawn with the R package "corrplot" version 0.84 (T. Wei and V. Simko, 2017, https://github.com/taiyun/corrplot). The diagnostic accuracy statistics were performed with the R package "pROC" version 1.1.00 (X. Robin et al., 2011, https://web.expasy.org/pROC/).

\section{Results}

Patients

Thirty-two consecutive patients met the inclusion criteria during the study period. A diagram depicting the flow of patients is provided in Additional file 2. Two patients could not be included because of the absence of investigators, four patients because of insufficient image quality, and one patient because of a severe tricuspid regurgitation.

Twenty-five patients were analyzed. Admission diagnosis was acute heart failure in 11 patients and septic shock in 14 patients. The median delay between admission and insertion of PAC was 3 [2-4] days.

\section{Clinical and hemodynamic characteristics}

A reduced RVEF was present in 16 patients (64\%). At the time of evaluation, $24 / 25$ patients were ventilated, $22 / 25$ received norepinephrine at a median dose of $0.29 \mu \mathrm{g} / \mathrm{kg} /$ min [0.14-0.50], and $4 / 25$ received dobutamine at a median dose of $5 \mu \mathrm{g} / \mathrm{kg} / \mathrm{min}$ [5-5]. The median Sequential Organ Failure Assessment score was 12 [10-14]. Mortality at day 28 was $56 \%$. RV free-wall thickness could not be measured for 9 patients, FAC could not be obtained for 2 patients, and EDDr could not be obtained for one patient. Clinical and hemodynamic characteristics of patients are reported respectively in Tables 1 and 2 .

\section{Correlations between echocardiographic indices of right ventricular systolic function and right ventricular ejection fraction}

Results of Spearman's correlations between TTE and continuous volumetric PAC parameters of RV systolic function are shown in Figs. 1 and 2. Correlation matrix of all RV echocardiographic indices and PAC variables is presented in the supplementary material (Additional file 3).

When compared to RVEF, TAPSE had the highest correlation coefficient (rho $=0.78,95 \%$ CI 0.52 to $0.89, p<0.001$ ). $\mathrm{S}^{\prime}$ was also correlated to RVEF (rho $=0.64,95 \%$ CI 0.60 to $0.80, p=0.001$ ) whereas FAC, RIMP, IVA, and EDDr did not (respectively rho $=0.18,95 \% \mathrm{CI}-0.24$ to $0.56, p=0.399$; rho $=-0.24,95 \%$ CI -0.58 to $0.17, p=0.247$; rho $=0.14$, $95 \% \mathrm{CI}-0.17$ to $0.58, p=0.494$; rho $=-0.30,95 \% \mathrm{CI}-0.68$ to $0.03, p=0.158$ ).
Table 1 Characteristics of patients at the time of evaluation

\begin{tabular}{|c|c|}
\hline Characteristics & Values \\
\hline Age (years) & $70[57-80]$ \\
\hline Female gender & $10(40 \%)$ \\
\hline BMI $\left(\mathrm{kg} / \mathrm{m}^{2}\right)$ & 24 [21-29] \\
\hline \multicolumn{2}{|l|}{ Admission diagnosis } \\
\hline Acute heart failure & $11(44 \%)$ \\
\hline Septic shock & $14(66 \%)$ \\
\hline Charlson comorbidity index & $6[2-7]$ \\
\hline \multicolumn{2}{|l|}{ Comorbidities } \\
\hline HFrEF & $3(12 \%)$ \\
\hline HFpEF & $5(20 \%)$ \\
\hline COPD & $8(32 \%)$ \\
\hline Pulmonary hypertension & $6(24 \%)$ \\
\hline None & $11(44 \%)$ \\
\hline SAPS $\|$ & $57[46-65]$ \\
\hline SOFA score & $12[10-14]$ \\
\hline Mortality at day 28 & $14(56 \%)$ \\
\hline Time from ICU admission (days) & $3[2-4]$ \\
\hline Mechanical ventilation & $24(96 \%)$ \\
\hline Tidal volume $(\mathrm{mL} / \mathrm{kg})$ & $6.5[6.0-7.1]$ \\
\hline Plateau pressure $\left(\mathrm{cmH}_{2} \mathrm{O}\right)$ & $20[13-24]$ \\
\hline End-expiratory pressure $\left(\mathrm{cmH}_{2} \mathrm{O}\right)$ & $7[5-10]$ \\
\hline Hemodynamic support & $23(92 \%)$ \\
\hline Norepinephrine & $22(88 \%)$ \\
\hline Norepinephrine dose ( $\mu \mathrm{g} / \mathrm{kg} / \mathrm{min})$ & $0.29[0.14-0.50]$ \\
\hline Dobutamine & $4(16 \%)$ \\
\hline Dobutamine dose ( $\mu \mathrm{g} / \mathrm{kg} / \mathrm{min})$ & $5[5-5]$ \\
\hline
\end{tabular}

Values are median [interquartile range] and number (percentage) $B M I$ body mass index, HFrEF heart failure with reduced ejection fraction, HFPEF heart failure with preserved ejection fraction, COPD chronic obstructive pulmonary disease, SAPS simplified acute physiologic score, SOFA Sequential Organ Failure Assessment, ICU intensive care unit

When evaluating correlations amongst indices, we only found TAPSE and $\mathrm{S}^{\prime}$ to be significantly correlated (rho $=0.71,95 \%$ CI 0.37 to $0.84, p<0.001$ ).

\section{Diagnostic accuracy of reduced right ventricular ejection fraction}

The results are presented in Table 3. TAPSE lower than $16 \mathrm{~mm}, \mathrm{~S}^{\prime}$ lower than $11 \mathrm{~cm} / \mathrm{s}$, and EDDr higher than 1 were always associated with a reduced RVEF. The receiver operating characteristic curves of all indices of reduced RVEF are shown in the supplementary material (Additional file 4). The results with the lower threshold of RVEF $(<25 \%)$ are presented in the supplementary material (Additional file 5). 
Table 2 Hemodynamics at the time of evaluation

\begin{tabular}{|c|c|c|}
\hline Variables & Values & Missing data, $n(\%)$ \\
\hline \multicolumn{3}{|l|}{ Systemic hemodynamics } \\
\hline Heart rate (bpm) & 94 [82-105] & \\
\hline MAP $(\mathrm{mmHg})$ & 76 [71-85] & \\
\hline SAP $(m m H g)$ & 103 [98-138] & \\
\hline DAP $(\mathrm{mmHg})$ & $58[53-67]$ & \\
\hline $\mathrm{SvO}_{2}(\%)$ & $71[63-77]$ & \\
\hline Lactate (mmol/L) & $2.2[1.5-3.7]$ & \\
\hline \multicolumn{3}{|l|}{ PAC pressures } \\
\hline RAP $(\mathrm{mmHg})$ & $9[6-10]$ & \\
\hline $\mathrm{sPAP}(\mathrm{mmHg})$ & $39[31-44]$ & \\
\hline $\mathrm{dPAP}(\mathrm{mmHg})$ & 19 [15-25] & \\
\hline mPAP $(\mathrm{mmHg})$ & $25[21-31]$ & \\
\hline PCWP (mmHg) & $11[9-13]$ & \\
\hline $\mathrm{CPP}(\mathrm{mmHg})$ & $51[47-55]$ & \\
\hline \multicolumn{3}{|l|}{ PAC thermodilution } \\
\hline $\mathrm{CO}(\mathrm{L} / \mathrm{min})$ & $4.9[3.8-6.1]$ & \\
\hline $\mathrm{SV}(\mathrm{mL})$ & $51[38-77]$ & \\
\hline RVEDV (mL) & $201[178-222]$ & \\
\hline RVESV (mL) & 141 [109-158] & \\
\hline RVEF (\%) & $30[20-37]$ & \\
\hline Reduced RVEF & $16(64 \%)$ & \\
\hline \multicolumn{3}{|c|}{ Transthoracic echocardiography } \\
\hline $\mathrm{CO}(\mathrm{L} / \mathrm{min})$ & $4.7[3.8-6.0]$ & 0 \\
\hline $\mathrm{SV}(\mathrm{mL})$ & $49[36-66]$ & 0 \\
\hline LVEF (\%) & $50[35-60]$ & 0 \\
\hline FAC (\%) & 29 [23-40] & $2(8 \%)$ \\
\hline TAPSE (mm) & 19 [15-24] & 0 \\
\hline $\mathrm{S}^{\prime}(\mathrm{cm} / \mathrm{s})$ & $12[9-15]$ & 0 \\
\hline RIMP & $0.81[0.69-1.03]$ & 0 \\
\hline IVA $\left(\mathrm{m} / \mathrm{s}^{2}\right)$ & $2.1[1.5-3.6]$ & 0 \\
\hline EDDr & $0.81[0.74-0.94]$ & $1(4 \%)$ \\
\hline $\mathrm{sPAP}(\mathrm{mmHg})$ & $34[31-41]$ & 7 (28\%) \\
\hline Free wall thickness (mm) & $3[2-4]$ & $9(36 \%)$ \\
\hline
\end{tabular}

Values are median [interquartile range] and number (percentage). Reduced RVEF is defined as lower than 35\% (see the "Material and methods" section) $M A P$ mean arterial pressure, $S A P$ systolic arterial pressure, DAP diastolic arterial pressure, $\mathrm{SvO}_{2}$ mixed venous oxygen saturation, $v P A C$ pulmonary artery catheter, RAP right atrial pressure, SPAP systolic pulmonary arterial pressure, dPAP diastolic pulmonary arterial pressure, $M P A P$ mean pulmonary arterial pressure, $P C W P$ pulmonary capillary wedge pressure, $C P P$ coronary perfusion pressure, $C O$ cardiac output, SV stroke volume, RVEDD right ventricle enddiastolic volume, RVESV right ventricle end-systolic volume, RVEF right ventricular ejection fraction, $L V E F$ left ventricular ejection fraction, $F A C$ fractional area change, TAPSE tricuspid annular plane systolic excursion, $S^{\prime}$ pic systolic velocity of pulsed tissue Doppler at tricuspid annular, RIMP right ventricular index of myocardial performance, IVA isovolumic acceleration, EDDr end-diastolic diameter ratio

\section{Discussion}

In this monocenter study with a limited number of patients, we found that amongst indices of right ventricular systolic function only TAPSE and S' were well correlated with and could therefore represent good surrogates of thermodilution-derived RVEF in severe critically ill patients.

The recommended method to evaluate RV systolic function is the RVEF measured with cardiac MRI [2]; however, cardiac MRI is difficult to perform in critically ill patients with ventilatory and hemodynamic supports. None of the indices of RV systolic function has been validated against RVEF in critically ill patients [9]. To investigate the relationship between these indices and RVEF in severe patients, we chose to use the RVEF derived from the washout portion of the thermodilution curve of a continuous volumetric PAC as reference method [19]. This is a limitation because it is not the gold standard for RVEF, and its accuracy has been debated [26]. It has been shown not to be interchangeable with cardiac MRI in some study of cardiology patients $[27,28]$ but to have good correlation in another [29]. In ICU patients, our population of interest, it has been historically validated against radio-nuclear angiography [30-33] and considered accurate to monitor patients with septic shock [34]. In the most recent studies in critically ill patients, when compared to three-dimensional transesophageal echocardiography, the reported bias was considered as clinically acceptable [24, 35]. Some studies [24, 28, 29, 35] report a systematic underestimation of the RVEF with thermodilution, a well-known technical limitation [25]. However, this limitation is unlikely to significantly impact our main results because we investigated echocardiographic indices as continuous physiological variables and their correlations with RVEF rather than predictors of true values. In addition, assuming that PAC may underestimate RVEF, we tested a lower cutoff value for the definition of reduced RVEF. It did not significantly modify our results. Another limitation could be the presence of TR. Severe TR was an exclusion criterion, but mild or moderate TR was present in a significant number of patients in our study. However, the continuous thermodilution has less limits than the cold bolus method, especially regarding dysrhythmias and TR [36, 37].

One strength of our study is that the risk of error with any of the compared method is minimized since our team has a long experience of PAC use and is well aware of its pitfalls and limitations, and TTEs were only performed by a limited number of trained experts with homogenous practices. Good intra- and interobserver variabilities are reported when measuring parameters of RV systolic function [38]. However, we did not specifically evaluate these variabilities in our study, which is a limitation. 


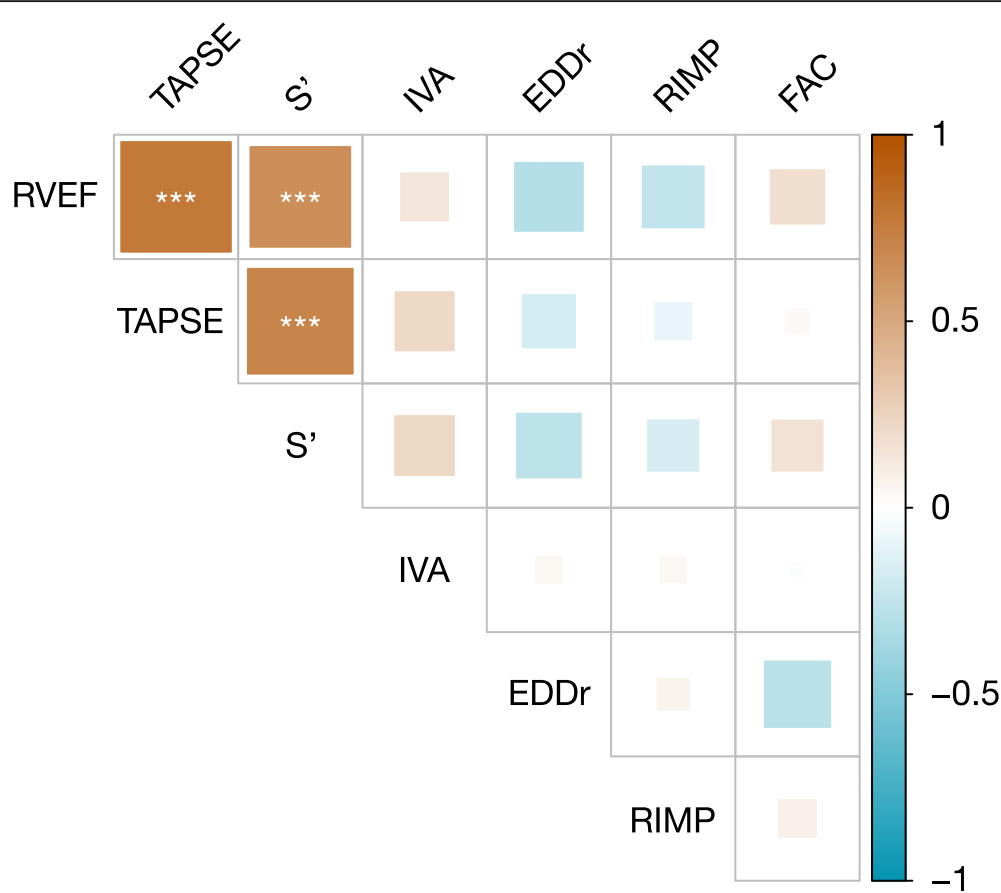

Fig. 1 Correlation matrix of transthoracic echocardiography parameters of right ventricular systolic function parameters and right ventricular ejection fraction. RVEF, right ventricular ejection fraction; TAPSE, tricuspid annular plane systolic excursion; S', peak systolic velocity of pulsed tissue Doppler at tricuspid annulus; IVA, isovolumic acceleration; EDDr, end-diastolic diameter ratio; RIMP, right ventricular index of myocardial performance; FAC, fractional area change. Spearman's correlations are computed amongst all variables. Positive correlations are represented by red squares and negative correlations by blue squares. Larger squares and darker colors represent higher correlation coefficient. ${ }^{* * *} p<0.001$, ${ }^{* *} p<0.01,{ }^{*} p<0.05$

Echocardiographic indices are usually proposed as a binary approach of RV dysfunction [8, 9]. Our study shows that TAPSE and $\mathrm{S}^{\prime}$, because of their good correlation with RVEF, could be reasonably used as continuous surrogates of RVEF and not only as a dichotomized marker of dysfunction. Thus, our results suggest that TAPSE and 'S' could therefore be valuable indicators for monitoring the evolution of the RVEF in ICU patients, but it has to be confirmed by a trending study. In addition, they both provide a valuable information when their values are lower than the recommended thresholds (TAPSE $<16 \mathrm{~mm}$ or $\mathrm{S}^{\prime}<10 \mathrm{~cm} / \mathrm{s}$ ) because it can confirm a clinical suspicion of RV dysfunction with a high PPV. However, a value above these cutoffs could not exclude an abnormal RVEF. TAPSE and S' are also well correlated with each other, which is consistent with previous literature [39]. The close relationship between these two parameters is not surprising since both are obtained from the displacement of the lateral part of the tricuspid annular plane. TAPSE and S' are thought to be indices of RV longitudinal contraction only, missing free wall abnormalities commonly encountered in acute pulmonary hypertension [40]. In our study, this limitation does not seem to have had a significant impact on the correlation with RVEF. However, due to technical limitation of the echocardiograph we used, we could not measure RV regional dysfunction with free-wall speckletracking strain in our study. This could be the subject of a further study.

FAC is known to be well correlated with RVEF in a recent meta-analysis of cardiology studies [6]. In a recent study comparing MRI-based RVEF to echocardiographic indices in non-critically ill patients, FAC appeared to correlate with RVEF better than TAPSE [7]. FAC has the theoretical advantage to be a global index of RV function, incorporating both longitudinal and free-wall contractility [9]. Thus, FAC or speckle-tracking strain may give additional information to TAPSE in clinical situations with acute pulmonary hypertension with free-wall abnormalities $[8,41,42]$. However, the feasibility of FAC has been reported around $60 \%$ in ventilated critically ill patients, mainly limited by insufficient acoustic window [39], and its interobserver agreement has been questioned [40]. In our study, despite exclusion of 4 patients because of insufficient acoustic window, the investigators were unable to measure the free wall thickness in a substantial number of patients and finally decided not to measure FAC in two patients. This technical limitation might be responsible for insufficient accuracy in the measurement of FAC in the setting of ventilated 

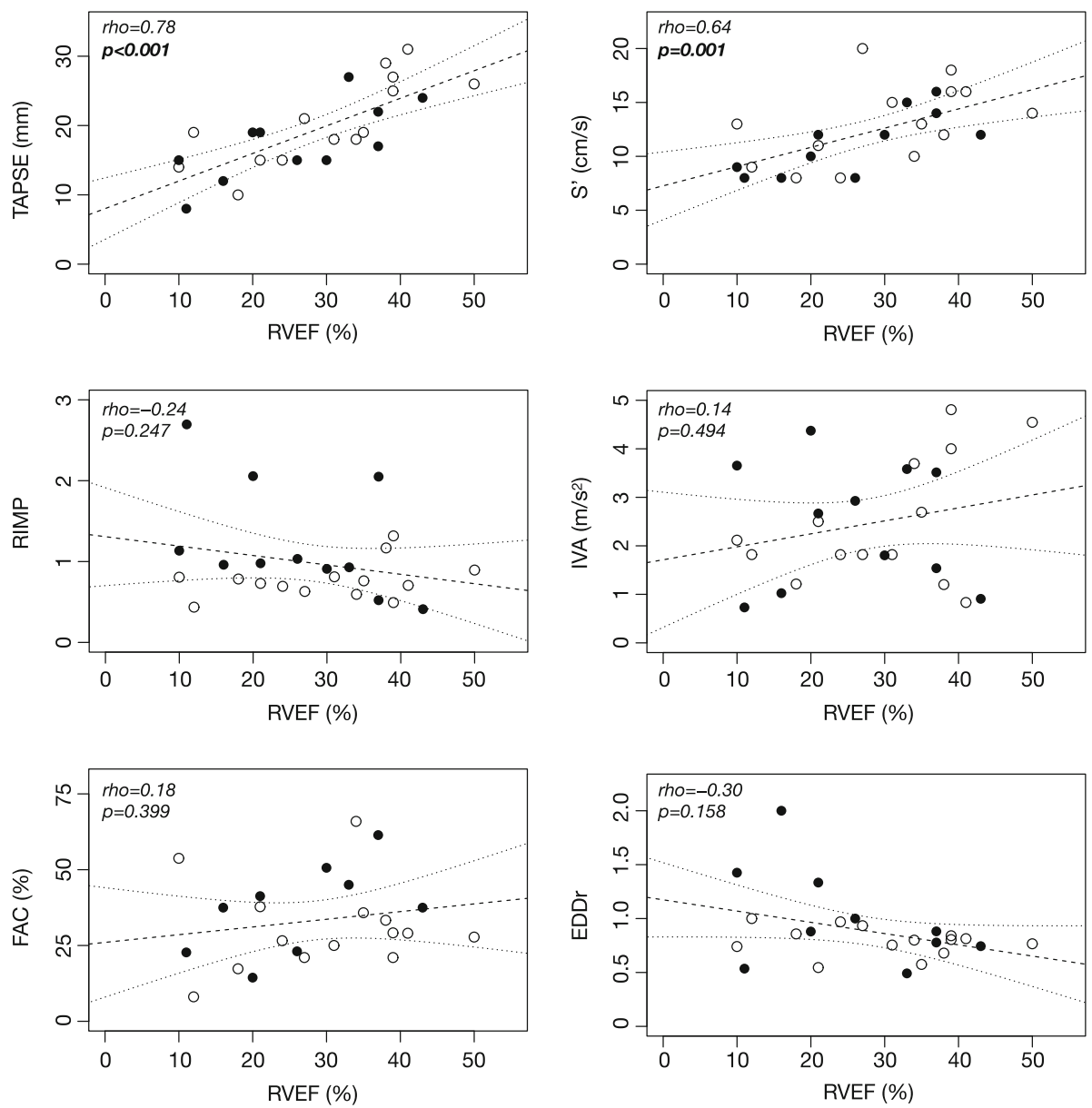

Fig. 2 Correlations between right ventricular ejection fraction and the main echocardiographic parameters of right ventricular function. RVEF, right ventricular ejection fraction; TAPSE, tricuspid annular plane systolic excursion; S', peak systolic velocity of pulsed tissue Doppler at tricuspid annulus; RIMP, right ventricular index of myocardial performance; IVA, isovolumic acceleration; EDDr, end-diastolic diameter ratio; RIMP, right ventricular index of myocardial performance; FAC, fractional area change. Symbol represents admission category: solid circles $(\bullet)$ are for acute heart failure and hollow circles (o) for septic shock. Dashed lines represent the regression lines and dotted lines the $95 \%$ confident interval of the regression lines. Rho and $p$ value are for Spearman's correlation

Table 3 Diagnostic accuracy of reduced right ventricular ejection fraction with echocardiographic indices at their recommended thresholds

\begin{tabular}{lllll}
\hline Indices & $S p$ & Se & NPV & PPV \\
\hline TAPSE $<16 \mathrm{~mm}$ & $100[100-100]$ & $56[31-81]$ & $56[45-75]$ & $100[100-100]$ \\
$S^{\prime}<10 \mathrm{~cm} / \mathrm{s}$ & $100[100-100]$ & $38[63-88]$ & $60[47-82]$ & $100[100-100]$ \\
RIMP $<0.55$ & $33[11-67]$ & $94[81-100]$ & $55[29-100]$ & $71[63-84]$ \\
IVA $<2.2 \mathrm{~m} / \mathrm{s}^{2}$ & $56[22-89]$ & $56[31-81]$ & $42[20-64]$ & $69[50-91]$ \\
FAC $<35 \%$ & $38[0-75]$ & $53[27-80]$ & $30[0-55]$ & $62[42-80]$ \\
EDDr $>1$ & $100[100-100]$ & $33[13-60]$ & $47[41-60]$ & $100[100-100]$
\end{tabular}

Reduced RVEF is defined as lower than 35\%. The tested threshold of the echocardiographic indices are those recommended in the guidelines [5]

$R V E F$ right ventricular ejection fraction, TAPSE tricuspid annular plane systolic excursion, $S^{\prime}$ pic systolic velocity of pulsed tissue Doppler at tricuspid annular, RIMP right ventricular index of myocardial performance, IVA isovolumic acceleration, FAC fractional area change, EDDr end-diastolic diameter ratio, Sp specificity, Se sensitivity, NPV negative predictive value, PPV positive predictive value 
critically ill patients and could participate in the poor performance of FAC in our study.

RIMP and IVA are thought to be relatively independent of loading conditions, and RIMP is known to encompass both systolic and diastolic components $[1,5]$. It is not the case of RVEF which depends on a complex and nonlinear relationship with preload and afterload [23, 43]. This could explain the absence of correlation with RVEF. However, RIMP and IVA do not correlate amongst them nor with any of the other measured parameters of RV function. What information is brought to physicians by these two indices in the critically ill patient remains to be explored.

EDDr was not correlated to RVEF as a continuous variable, but all patients with RV dilation on the TTE $(E D D r>1)$ had a reduced RVEF. However, a decrease in RVEF can be missed by a simple measure of RV dilation. This is expected as dilation of the RV is one of the determinants of reduced RVEF, but not the only one.

All other tested indices were neither correlated to RVEF nor efficient classifiers of reduced RVEF at the tested cutoffs. Thus, our study shows that amongst all the recommended indices of RV systolic function, TAPSE and $S^{\prime}$ are those who have the most determinants in common with RVEF. However, our results do not imply that other indices of RV function should not be used since they bring additional information to RVEF that might help the clinician when assessing RV systolic function.

The present study focuses on severe critically ill patients with a high mortality rate, high SAPS II and SOFA scores, elevated lactate level, and who required both vasopressors and mechanical ventilation while most of the available literature regarding validation of echocardiographic indices of RV systolic function with RVEF were performed on stable cardiology patients. The investigation of RV function in patients with refractory shock is clinically relevant because it is a subset of patient with poor outcome in which clinician should evoke the possibility of RV failure in the hemodynamic picture because it can modify the management of the supportive therapies (fluid balance, choice of vasoactive drugs, settings of mechanical ventilation) and potentially impact outcome $[1-3,44]$. In our cohort, a substantial number of patients had a low RVEF that could have been missed if it has not been investigated.

Our study has some limitations. It is a monocenter study with a limited number of patients. Thus, it is likely that we only identified the strongest correlations and cannot exclude that a larger study may reveal significant correlations between the other indices. We compared echocardiographic indices of RV systolic function to RVEF in patients with primary septic shock or acute heart failure in which poor response to initial therapy leads to the decision of PAC monitoring by the attending physician. These are already resuscitated patients who achieved standard hemodynamic targets but remain dependent on vasopressor and/or inotropes and do not recover from their organ failures or have persistent hyperlactatemia. None of our patients had myocardial infarction, and CPP did not appear to be limiting. It is a particular severe subtype of patients and represents a selection bias that prevents generalization of our results to all critically ill patients. These are preliminary data that advocate for a larger study to confirm our results. Another limitation is that these indices were not tested in patients with non-resuscitated shock or primary RV failure at ICU admission (pulmonary embolism with shock or RV infarction with shock). Our results should not be extrapolated to these clinical scenarios.

\section{Conclusion}

We found that amongst indices of right ventricular systolic function, TAPSE and $S^{\prime}$ were well correlated with thermodilution-derived RVEF in critically ill patients.

\section{Additional files}

Additional file 1: Criteria for severe tricuspid regurgitation and Figure of Doppler tissue velocities and time intervals obtained at lateral tricuspid valve annulus (DOCX $218 \mathrm{~kb}$ )

Additional file 2: Flow chart of the study (PDF $14 \mathrm{~kb}$ )

Additional file 3: Correlation matrix of continuous volumetric pulmonary arterial catheter and echocardiographic parameters of right ventricular function (DOCX $596 \mathrm{~kb}$ )

Additional file 4: Receiver operating characteristic curves of echocardiographic indices for diagnosis of reduced right ventricular ejection fraction (DOCX $160 \mathrm{~kb}$ )

Additional file 5: Diagnostic accuracy of reduced right ventricular ejection fraction (<25\%) with echocardiographic indices (DOCX 17 kb)

\section{Abbreviations}

Cl: Confident interval; CO: Cardiac output; dPAP: Diastolic pulmonary arterial pressure; EDDr: End-diastolic diameter ratio; ICU: Intensive care medicine;

IVA: Isovolumic acceleration; LV: Left ventricle; LVEF: Left ventricular ejection fraction; mPAP: Mean pulmonary arterial pressure; MRI: Magnetic resonance imaging; NPV: Negative predictive value; PAC: Pulmonary artery catheter; PAP: Pulmonary arterial pressure; PCWP: Pulmonary capillary wedge pressure; PPV: Positive predictive value; RAP: Right atrial pressure; RIMP: Right ventricular index of myocardial performance; RV: Right ventricle; RVEDV: Right ventricular end-diastolic volume; RVEF: Right ventricular ejection fraction; RVESV: Right ventricular end-systolic volume; SAPS: Simplified acute physiological score; Se: Sensitivity; SOFA: Sequential Organ Failure Assessment; Sp: Specificity; sPAP: Systolic pulmonary arterial pressure; SV: Stroke volume; TAPSE: Tricuspid annular plane systolic excursion; TR: Tricuspid regurgitation; TTE: Transthoracic echocardiography

\section{Acknowledgements}

Not Applicable

\section{Authors' contributions}

$\mathrm{RB}$ designed the study, participated in the collection of the data, performed the statistics, and participated in the interpretation of the data and drafting of the manuscript. XR and TJ participated in the collection of the data. BC 
and $\mathrm{AM}$ participated in the interpretation of the data and drafting of the manuscript. All authors read and approved the final manuscript.

\section{Funding}

None.

\section{Availability of data and materials}

The datasets used and/or analyzed during the current study are available from the corresponding author on reasonable request.

\section{Ethics approval and consent to participate}

This study was approved by an institutional ethics committee (Comité d'éthique de la Société Française d'Anesthésie-Réanimation, IRB 000102542016-034) which waived the need of signed informed consent.

\section{Consent for publication}

Not Applicable.

\section{Competing interests}

The authors declare that they have no competing interests.

\section{Author details}

'Department of Anaesthesia and Critical Care, Lariboisière Hospital, DMU Parabol, APHP.Nord, Paris, France. ${ }^{2}$ Inserm UMR-S942, Mascot, Paris, France. ${ }^{3}$ Université de Paris, Paris, France. ${ }^{4}$ Réanimation Chirurgical Polyvalente, Hôpital Lariboisière, 2 rue Ambroise Paré, 75475 Paris Cedex 10, France.

\section{Received: 24 April 2019 Accepted: 27 August 2019}

\section{Published online: 13 September 2019}

\section{References}

1. Harjola V-P, Mebazaa A, Čelutkienè J, Bettex D, Bueno H, Chioncel O, et al. Contemporary management of acute right ventricular failure: a statement from the Heart Failure Association and the Working Group on Pulmonary Circulation and Right Ventricular Function of the European Society of Cardiology. Eur J Heart Fail. 2016;18:226-41.

2. Konstam MA, Kiernan MS, Bernstein D, Bozkurt B, Jacob M, Kapur NK, et al. Evaluation and management of right-sided heart failure: a scientific statement from the American Heart Association. Circulation. 2018;137:e578-622.

3. Vieillard-Baron A, Naeije R, Haddad F, Bogaard HJ, Bull TM, Fletcher N, et al Diagnostic workup, etiologies and management of acute right ventricle failure : a state-of-the-art paper. Intensive Care Med. 2018:44:774-90.

4. Cecconi M, De Backer D, Antonelli M, Beale R, Bakker J, Hofer C, et al. Consensus on circulatory shock and hemodynamic monitoring. Task force of the European Society of Intensive Care Medicine. Intensive Care Med. 2014;40:1795-815.

5. Rudski LG, Lai WW, Afilalo J, Hua L, Handschumacher MD, Chandrasekaran K, et al. Guidelines for the echocardiographic assessment of the right heart in adults: a report from the American Society of Echocardiography endorsed by the European Association of Echocardiography, a registered branch of the European Society of Cardiology, and the Canadian Society of Echocardiography. J Am Soc Echocardiogr. 2010;23:685-713.

6. Lee JZ, Low S-W, Pasha AK, Howe CL, Lee KS, Suryanarayana PG Comparison of tricuspid annular plane systolic excursion with fractional area change for the evaluation of right ventricular systolic function: a metaanalysis. Open Heart. 2018;5:e000667.

7. Simsek E, Nalbantgil S, Ceylan N, Zoghi M, Kemal HS, Engin C, et al. Assessment of right ventricular systolic function in heart transplant patients: correlation between echocardiography and cardiac magnetic resonance imaging. Investigation of the accuracy and reliability of echocardiography. Echocardiography. 2017:34:1432-8.

8. Focardi M, Cameli M, Carbone SF, Massoni A, De Vito R, Lisi M, et al. Traditional and innovative echocardiographic parameters for the analysis of right ventricular performance in comparison with cardiac magnetic resonance. Eur Heart J Cardiovasc Imaging. 2015;16:47-52.

9. Huang SJ, Nalos M, Smith L, Rajamani A, McLean AS. The use of echocardiographic indices in defining and assessing right ventricular systolic function in critical care research. Intensive Care Med. 2018:44:868-83.

10. Lancellotti P, Tribouilloy C, Hagendorff A, Popescu BA, Edvardsen T, Pierard $L A$, et al. Recommendations for the echocardiographic assessment of native valvular regurgitation: an executive summary from the European
Association of Cardiovascular Imaging. Eur Heart J Cardiovasc Imaging. 2013;14:611-44.

11. Bennett D, Boldt J, Brochard L, Coriat P, Dhainaut J-F, Edwards D, et al. Expert Panel: The use of the pulmonary artery catheter. Intensive Care Med. 1991;17:I-VIII.

12. Teboul J-L, Besbes $M$, Andrivet $P$, Axler $O$, Douguet $D$, Zelter M, et al. A bedside index assessing the reliability of pulmonary artery occlusion pressure measurements during mechanical ventilation with positive endexpiratory pressure. J Crit Care. 1992;7:22-9.

13. Yelderman M. Continuous measurement of cardiac output with the use of stochastic system identification techniques. J Clin Monit. 1990;6:322-32.

14. Siegel LC, Hennessy MM, Pearl RG. Delayed time response of the continuous cardiac output pulmonary artery catheter. Anesth Analg. 1996:83:1173-7.

15. Hamilton W, Moore J, Kinsman J, Spurling RG. Studies on the circulation IV. Further analysis of the injection method, and of changes in hemodynamtcs under physiological and pathological conditions. Am J Phys. 1932;99:534-51.

16. Stewart GN. Researches on the circulation time and on the influences which affect it. J Physiol. 1897;22:159-83.

17. Fegler G. Measurement of cardiac output in anaesthetized animals by a thermodilution method. Q J Exp Physiol Cogn Med Sci. 1954;39:153-64.

18. Ganz W, Donoso R, Marcus HS, Forrester JS, Swan HJ. A new technique for measurement of cardiac output by thermodilution in man. Am J Cardiol. 1971;27:392-6.

19. Rapaport E, Wong M, Ferguson RE, Bernstein P, Wiegand BD. Right ventricular volumes in patients with and without heart failure. Circulation. 1965;31:531-41.

20. Kay HR, Afshari M, Barash P, Webler W, Iskandrian A, Bemis C, et al. Measurement of ejection fraction by thermal dilution techniques. J Surg Res. 1983:34:337-46.

21. Mercado P, Maizel J, Beyls C, Kontar L, Orde S, Huang S, et al. Reassessment of the accuracy of cardiac Doppler pulmonary artery pressure measurements in ventilated ICU patients: a simultaneous Dopplercatheterization study. Crit Care Med. 2019;47:41-8.

22. Pavlicek M, Wahl A, Rutz T, de Marchi SF, Hille R, Wustmann K, et al. Right ventricular systolic function assessment: rank of echocardiographic methods vs. cardiac magnetic resonance imaging. Eur J Echocardiogr. $2011 ; 12: 871-80$

23. Vanderpool RR, Rischard F, Naeije R, Hunter $K$, Simon MA. Simple functional imaging of the right ventricle in pulmonary hypertension: can right ventricular ejection fraction be improved? Int J Cardiol. 2016;223:93-4.

24. Zink W, Nöll J, Rauch H, Bauer H, Desimone R, Martin E, et al. Continuous assessment of right ventricular ejection fraction: new pulmonary artery catheter versus transoesophageal echocardiography. Anaesthesia. 2004:59:1126-32.

25. Maruschak GF, Schauble JF. Limitations of thermodilution ejection fraction: degradation of frequency response by catheter mounting of fast-response thermistors. Crit Care Med. 1985;13:679-82.

26. Leibowitz AB. Pulmonary artery catheter determined right ventricular ejection fraction and right ventricular end-diastolic volume: another case of "The Emperor Has No Clothes.". Crit Care Med. 2009;37:2992.

27. Fujiwara S, Motoki K, Oshika H, Tomobuchi Y, Ueno Y, Nishio I. Assessment of right ventricular function by magnetic resonance imaging of old myocardial infarction. J Cardiol. 1995;26:203-11.

28. Hoeper MM, Tongers J, Leppert A, Baus S, Maier R, Lotz J. Evaluation of right ventricular performance with a right ventricular ejection fraction thermodilution catheter and MRI in patients with pulmonary hypertension. Chest. 2001;120:502-7.

29. Globits S, Pacher R, Frank H, Pacher B, Mayr H, Neuhold A, et al. Comparative assessment of right ventricular volumes and ejection fraction by thermodilution and magnetic resonance imaging in dilated cardiomyopathy. Cardiology. 1995;86:67-72.

30. Voelker W, Gruber HP, Ickrath O, Unterberg R, Karsch KR. Determination of right ventricular ejection fraction by thermodilution technique--a comparison to biplane cineventriculography. Intensive Care Med. 1988; 14(Suppl 2):461-6.

31. Urban P, Scheidegger D, Gabathuler J, Rutishauser W. Thermodilution determination of right ventricular volume and ejection fraction: a comparison with biplane angiography. Crit Care Med. 1987;15:652-5.

32. Dhainaut JF, Brunet F, Monsallier JF, Villemant D, Devaux JY, Konno M, et al. Bedside evaluation of right ventricular performance using a rapid computerized thermodilution method. Crit Care Med. 1987;15:148-52. 
33. Vincent IL, Thirion M, Brimioulle S, Lejeune P, Kahn RJ. Thermodilution measurement of right ventricular ejection fraction with a modified pulmonary artery catheter. Intensive Care Med. 1986;12:33-8.

34. Vincent JL, Reuse C, Frank N, Contempré B, Kahn RJ. Right ventricular dysfunction in septic shock: assessment by measurements of right ventricular ejection fraction using the thermodilution technique. Acta Anaesthesiol Scand. 1989;33:34-8.

35. De Simone R, Wolf I, Mottl-Link S, Böttiger BW, Rauch H, Meinzer H-P, et al. Intraoperative assessment of right ventricular volume and function. Eur J Cardiothorac Surg. 2005;27:988-93.

36. Reuter DA, Huang C, Edrich T, Shernan SK, Eltzschig HK. Cardiac output monitoring using indicator-dilution techniques: basics, limits, and perspectives. Anesth Analg. 2010;110:799-811.

37. Buffington CW, Nystrom EUM. Neither the accuracy nor the precision of thermal dilution cardiac output measurements is altered by acute tricuspid regurgitation in pigs. Anesth Analg. 2004;98:884-90 table of contents.

38. Ling LF, Obuchowski NA, Rodriguez L, Popovic Z, Kwon D, Marwick TH. Accuracy and interobserver concordance of echocardiographic assessment of right ventricular size and systolic function: a quality control exercise. J Am Soc Echocardiogr. 2012;25:709-13.

39. Fichet J, Moreau L, Genée O, Legras A, Mercier E, Garot D, et al. Feasibility of right ventricular longitudinal systolic function evaluation with transthoracic echocardiographic indices derived from tricuspid annular motion: a preliminary study in acute respiratory distress syndrome. Echocardiography. 2012;29:513-21.

40. Wright L, Dwyer N, Power J, Kritharides L, Celermajer D, Marwick TH. Right ventricular systolic function responses to acute and chronic pulmonary hypertension: assessment with myocardial deformation. J Am Soc Echocardiogr. 2016;29:259-66.

41. Fukuda $Y$, Tanaka H, Sugiyama D, Ryo $K$, Onishi T, Fukuya $H$, et al. Utility of right ventricular free wall speckle-tracking strain for evaluation of right ventricular performance in patients with pulmonary hypertension. J Am Soc Echocardiogr. 2011;24:1101-8.

42. Meris A, Faletra F, Conca C, Klersy C, Regoli F, Klimusina J, et al. Timing and magnitude of regional right ventricular function: a speckle tracking-derived strain study of normal subjects and patients with right ventricular dysfunction. J Am Soc Echocardiogr. 2010;23:823-31.

43. Vonk Noordegraaf $\mathrm{A}$, Westerhof $\mathrm{BE}$, Westerhof $\mathrm{N}$. The relationship between the right ventricle and its load in pulmonary hypertension. J Am Coll Cardiol. 2017;69:236-43.

44. King C, May CW, Williams J, Shlobin OA. Management of right heart failure in the critically ill. Crit Care Clin. 2014;30:475-98.

\section{Publisher's Note}

Springer Nature remains neutral with regard to jurisdictional claims in published maps and institutional affiliations.

Ready to submit your research? Choose BMC and benefit from:

- fast, convenient online submission

- thorough peer review by experienced researchers in your field

- rapid publication on acceptance

- support for research data, including large and complex data types

- gold Open Access which fosters wider collaboration and increased citations

- maximum visibility for your research: over $100 \mathrm{M}$ website views per year

At BMC, research is always in progress.

Learn more biomedcentral.com/submissions 